\title{
Insulin-induced cortical actin remodeling promotes GLUT4 insertion at muscle cell membrane ruffles
}

\author{
Peter Tong, ${ }^{1}$ Zayna A. Khayat, ${ }^{1,2}$ Carol Huang, ${ }^{1}$ Nish Patel, ${ }^{1}$ Atsunori Ueyama, \\ and Amira Klip ${ }^{1,2}$
}

${ }^{1}$ Programme in Cell Biology, The Hospital for Sick Children, Toronto, Canada

${ }^{2}$ Department of Biochemistry, University of Toronto, Toronto, Canada

Address correspondence to: Amira Klip, The Hospital for Sick Children, Programme in Cell Biology,

555 University Avenue, Toronto, Ontario, Canada M5G 1X8.

Phone: (416) 813-6392; Fax: (416) 813-5028; E-mail: amira@sickkids.on.ca.

Peter Tong's present address is: Department of Medicine and Therapeutics, Chinese University of Hong Kong, Prince of Wales Hospital, Shatin, NT, Hong Kong, China.

Atsunori Ueyama's present address is: Division of Molecular Genetics, Institute for Enzyme Research, University of Tokushima, Tokushima, Japan.

Peter Tong and Zayna A. Khayat contributed equally to this work.

Received for publication January 25, 2001, and accepted in revised form June 26, 2001.

Insulin stimulates glucose uptake by recruiting glucose transporter 4 (GLUT4) from an intracellular compartment to the cell surface; this phenomenon is defective in type 2 diabetes. Here we examine the involvement of actin filaments in GLUT4 translocation and their possible defects in insulin resistance, using L6 myotubes expressing myc-tagged GLUT4. Insulin caused membrane ruffling, a dynamic distortion of the myotube dorsal surface. Fluorescence microscopy and immunogold staining of surface GLUT4myc coupled to backscatter electron microscopy revealed a high density of this protein in membrane ruffles. The t-SNAREs syntaxin 4 and SNAP-23 were also abundant in these regions. Below the membrane, GLUT4 and the vesicular protein VAMP2, but not VAMP3, colocalized with the actin structures supporting the membrane ruffles. GLUT4myc externalization and membrane ruffles were reduced by jasplakinolide and by swinholide-A, drugs that affect actin filament stability and prevent actin branching, respectively. Insulin resistance generated by prolonged ( 24 hours) exposure of myotubes to high glucose and insulin diminished the acute insulin-dependent remodeling of cortical actin and GLUT4myc translocation, reminiscent of the effect of swinholide-A. We propose that GLUT4 vesicle incorporation into the plasma membrane involves insulin-dependent cortical actin remodeling and that defective actin remodeling contributes to insulin resistance.

J. Clin. Invest. 108:371-381 (2001). DOI:10.1172/JCI200112348.

\section{Introduction}

The regulation of glucose uptake by insulin is critical for the maintenance of glucose homeostasis. The uptake of glucose into skeletal muscle, a major insulinresponsive organ, is mediated by the insulin-responsive glucose transporter, GLUT4. GLUT4-containing vesicles are largely sequestered intracellularly, and an insulin-derived signal(s) triggers their recruitment to the muscle plasma membrane to increase glucose influx $(1,2)$. This phenomenon is abnormally diminished in type 2 diabetes, resulting in insulin resistance (3-5). The mechanism by which insulin directs the insertion of GLUT4 proteins into the plasma membrane is not fully understood. Moreover, the cellular basis of insulin resistance remains poorly explained. It is well recognized that insulin causes a rapid and marked remodeling of actin filaments below the plasma membrane, promoting membrane ruffling in cells as diverse as myotubes (6), adipocytes $(7,8)$, and fibroblasts (9-11). Our previous studies in L6 skeletal muscle cells suggest that actin remodeling after insulin stimu- lation expedites the delivery of signaling molecules to specific cellular loci for interactions with downstream targets including GLUT4-containing vesicles (12). Moreover, we and others have shown that disruption of the actin cytoskeleton prevents the arrival of GLUT4 at the cell surface of muscle and adipose cells $(6,8,13-15)$. The exact role of the actin cytoskeleton in the insertion of GLUT4 at the plasma membrane is not known and is the basis for this study.

The proteins that regulate the fusion of GLUT4-containing vesicles with the plasma membrane are isoforms of those constituting the well-characterized fusion machinery of synaptic vesicles in neuroendocrine cells, termed SNARE (soluble $N$-ethylmaleimide sensitive factor attachment protein receptor) proteins. The SNARE hypothesis proposes that interaction between proteins resident on vesicles ( $v$-SNARE) and on the target membrane (t-SNARE) promotes fusion of the two membranes (16). Recent studies have suggested that the incorporation of insulin-responsive GLUT4-containing 
vesicles into the plasma membrane of muscle and adipose cells involves the v-SNARE protein isoform vesicle associated membrane protein 2 (VAMP2, but not the related VAMP3/cellubrevin isoform) (17-19) and the t-SNAREs syntaxin $4(20,21)$ and synaptosome-associated 23-kDa protein (SNAP-23) $(22,23)$. Whether these SNARE proteins link to the actin cytoskeleton during the fusion of GLUT4 vesicles with the plasma membrane is not known.

Here we explore the hypothesis that cortical actin remodeling participates in the recruitment of GLUT4containing vesicles to the plasma membrane. We further hypothesize that defects in the regulation of the actin cytoskeleton may underlie some forms of insulin resistance. L6 myotubes, which differentiate in vitro from myoblasts into multinucleated myotubes by multiple cell fusions, contain a large cytoplasmic space rich in actin filaments, which is amenable to morphological analysis at the light microscopy level. The results presented suggest that insulin recruits intracellular GLUT4 and VAMP2 to specific loci in the plasma membrane where ruffles occur. Insulin-dependent remodeling of the actin cytoskeleton leads to ruffle formation where the t-SNAREs, syntaxin 4 and SNAP-23, concentrate. Importantly, interfering with actin remodeling by either specific pharmacological agents or by inducing insulin resistance with high glucose and insulin abrogates membrane ruffle formation and largely prevents GLUT4 insertion into the plasma membrane in response to insulin.

\section{Methods}

Reagents. Jasplakinolide, swinholide-A, Oregon Greenphalloidin, and FITC-conjugated concanavalin-A were purchased from Molecular Probes Inc. (Eugene, Oregon, USA). O-phenylenediamine dihydrochloride (OPD) reagent and $m A b$ to $\beta$-actin were purchased from Sigma-Aldrich (Oakville, Ontario, Canada). An mAb against myc (9E10) was obtained from Santa Cruz Biotechnology Inc. (Santa Cruz, California, USA). Polyclonal antibodies to VAMP2, VAMP3, syntaxin 4 , and SNAP-23 were generated as described previously $(8,24)$. FITC-conjugated goat anti-mouse, FITC-conjugated goat-anti-rabbit, Cyanine 3-conjugated goat anti-mouse (Cy3-conjugated goat antimouse), Cy3-conjugated goat anti-rabbit, and 30-nm gold-conjugated goat anti-mouse antibodies were obtained from Jackson Immunoresearch Laboratories Inc. (West Grove, Pennsylvania, USA).

Cell culture. L6 muscle cells expressing c-myc epitope tagged GLUT4 (GLUT4myc) $(25,26)$ were maintained in myoblast monolayer culture in $\alpha$-MEM containing $10 \%$ (vol/vol) FBS and $1 \%(\mathrm{vol} / \mathrm{vol})$ antibiotic-antimycotic solution $(10,000 \mathrm{U} / \mathrm{ml}$ penicillin $\mathrm{G}, 10 \mathrm{mg} / \mathrm{ml}$ streptomycin, and $25 \mathrm{mg} / \mathrm{ml}$ amphotericin B) in an atmosphere of $5 \% \mathrm{CO}_{2}$ at $37^{\circ} \mathrm{C}$. Cells were subcultured by trypsinization of subconfluent cultures using $0.25 \%$ ( $\mathrm{vol} / \mathrm{vol}$ ) trypsin. For differentiation into myotubes, myoblasts were plated in medium containing $2 \%$ (vol/vol) FBS at
$10^{4}$ cells $/ \mathrm{ml}$ to allow spontaneous myoblast fusion. Medium was changed every 48 hours. Myotubes were ready for experimentation 6-8 days after seeding.

Fluorescence, confocal and scanning electron microscopy. For fluorescence microscopy, L6 GLUT4myc myotubes, grown on 25 -mm-diameter glass coverslips, were deprived of serum for 4 hours and treated with $100 \mathrm{nM}$ insulin for up to 30 minutes at $37^{\circ} \mathrm{C}$. Labeling of actin filaments with fluorophore-coupled phalloidin and immunostaining of specific antigens in fixed and permeabilized myotubes were carried out as described previously (12). The primary antibody dilution factors were as follows: myc, 1:150; VAMP-2, 1:100; VAMP-3, 1:100; syntaxin 4, 1:150; and SNAP-23, $1: 150$ in $0.1 \%$ (wt/vol) BSA/PBS. To label actin filaments simultaneously, fixed and permeabilized cells were incubated for 1 hour at room temperature with Oregon Green-phalloidin (0.01 U/coverslip) during the incubation with secondary antibody. GLUT4myc translocation was determined by cell-surface immunostaining of intact myotubes (26). For fluorescence and confocal microscopy, cells were examined with either an inverted Leica DM-IRB microscope equipped with a Leica confocal laser scanning imaging system (TCS 4D) or a Zeiss LSM 510 laser scanning confocal microscope (Carl Zeiss, Inc. Don Mills, Ontario, Canada).

For scanning electron microscopy and immunogold labeling, control and insulin-stimulated L6-GLUT4myc myotubes were fixed with $2 \%(\mathrm{vol} / \mathrm{vol})$ paraformaldehyde and $0.2 \%$ (vol/vol) glutaraldehyde in phosphate buffer for 30 minutes before immunolabeling with primary anti-myc antibody and secondary goat anti-mouse antibody linked to $30 \mathrm{~nm}$-diameter gold particles. The cells were dehydrated in graded ethanol and were critically dried. Specimens were coated with carbon and examined on a JEOL JSM 820 scanning electron microscope. Composite signals of the scanning mode and the backscattered electrons from the gold particles were acquired simultaneously. The density of gold particles was calculated as follows: A $3 \times 3 \mathrm{~cm}$ grid was applied to each image (corresponding scale of the image $1 \mathrm{~cm}=1$ $\mu \mathrm{m}$ of cell surface). GLUT4 particles were counted in five squares from unstimulated cells, ruffled areas in insulin-treated cells or nonruffled areas in insulin-treated cells. Image J, a public-domain Java image-processing program inspired by NIH Image software, was used to analyze the grids. SPSS software (version 10; SPSS Inc., Chicago, Illinois, USA) was used for statistical analysis.

Densitometric assay of surface GLUT4myc. After serum deprivation, $\mathrm{L} 6$ myotubes were left untreated or treated with $100 \mathrm{nM}$ insulin for various times at $37^{\circ} \mathrm{C}$. After this period, cells were washed three times with ice-cold PBS on ice followed by blocking with $5 \%$ (vol/vol) goat serum in PBS for 10 minutes. Cells were then incubated with anti-myc mAb in HEPES-buffered RPMI containing 3\% (vol/vol) goat serum for 60 minutes at $4{ }^{\circ} \mathrm{C}$ before fixation with $3 \%$ (vol/vol) formaldehyde in PBS for 3 minutes. Cells were incubated with $100 \mathrm{mM}$ glycine in PBS at $4^{\circ} \mathrm{C}$ for 10 minutes followed by incu- 
a

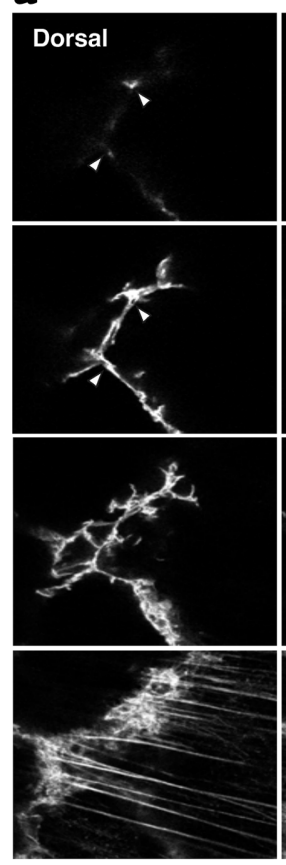

F-Actin

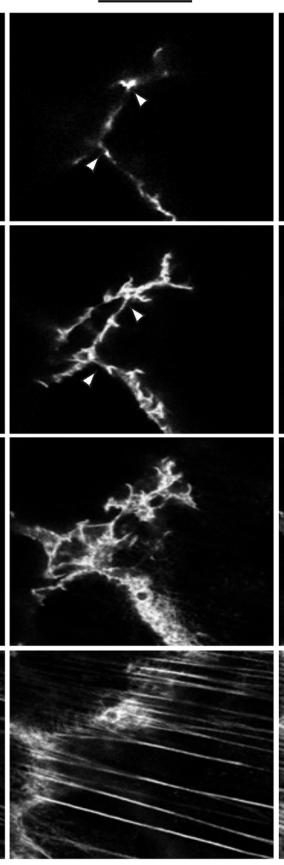

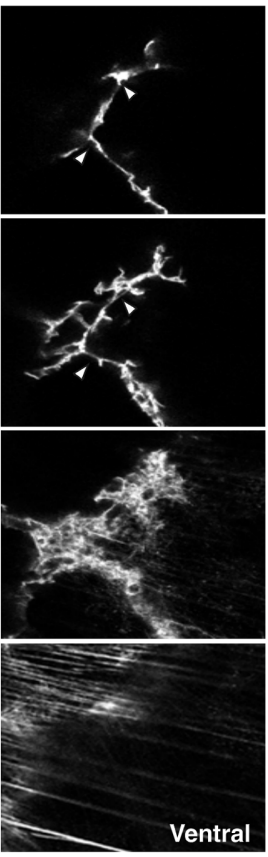

b
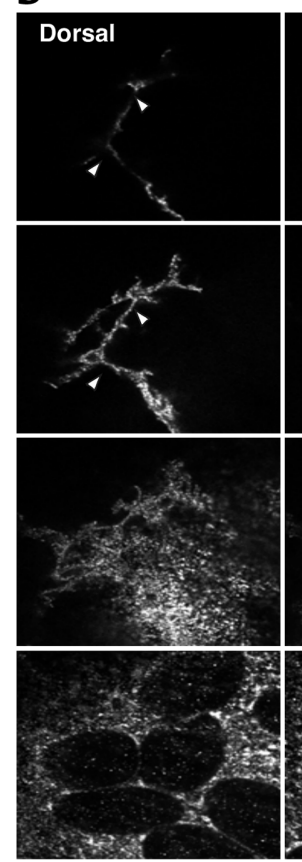

GLUT4-myc
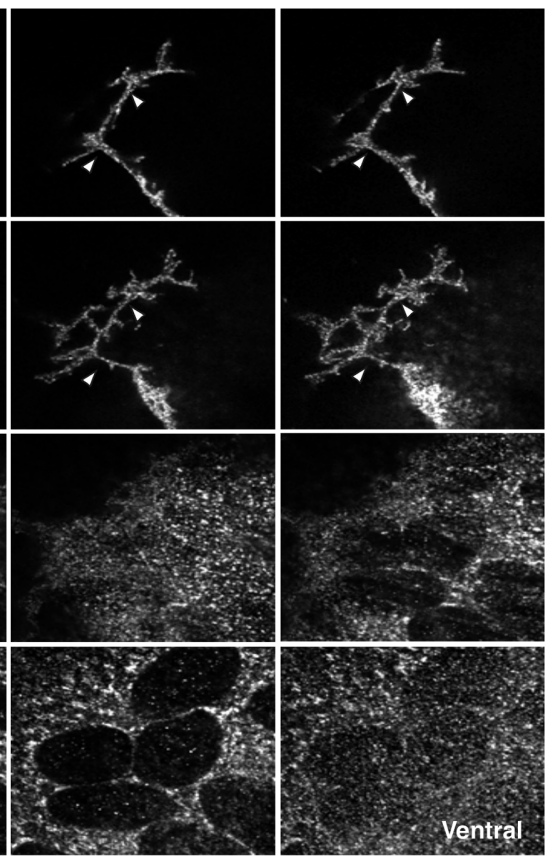

\section{Figure 1}

Confocal scanning series of GLUT4 colocalizing with newly formed dorsal actin-rich structures in response to insulin. Serum-deprived L6 GLUT4myc myotubes were treated with or without $100 \mathrm{nM}$ insulin for 10 minutes at $37^{\circ} \mathrm{C}$. Afterward, the cells were fixed, permeabilized, and double-stained for actin (Oregon Green-phalloidin) and GLUT4myc (anti-myc antibody followed by Cy3-conjugated secondary antibody) as described in Methods. Serial optical sections of $0.67 \mu \mathrm{m}$ thickness along the $z$-axis were generated from myotubes as described in Methods. Optical slices taken from the dorsal to the ventral cell surface of myotubes show filamentous actin (a) and myc (b) after 10 minutes of $100 \mathrm{nM}$ insulin treatment. The multiple myonuclei (b, dark ovals) were identified in parallel experiments by DAPI staining (data not shown). The progression of images is linear from left to right. Scale: $5 \mathrm{~mm}$ in image is equivalent to $10 \mu \mathrm{m}$ in specimen. White arrows indicate regions of remodeled actin filaments. The images are representative of five experiments.

bation with horseradish peroxidase-conjugated (HRPconjugated) goat anti-mouse IgG $(1: 1,000)$ in PBS containing $3 \%$ goat serum for 60 minutes. To quantitate the amount of bound antibody, OPD reagent was added at room temperature for up to 30 minutes and the reaction stopped by adding $3 \mathrm{~N}$ hydrochloric acid. An aliquot of the reaction was removed for measuring the absorbance at $492 \mathrm{~nm}(26)$.

$2-\left[{ }^{3} H\right]$ deoxyglucose uptake. After serum deprivation, L6 myotubes were left untreated or treated with 100 $\mathrm{nM}$ insulin for 30 minutes at $37^{\circ} \mathrm{C}$. After this period, cells were washed three times with glucose-free HEPES-buffered saline solution $(140 \mathrm{mM} \mathrm{NaCl}, 20$ mM Na-HEPES [pH 7.4], $2.5 \mathrm{mM} \mathrm{MgSO}_{4}, 5 \mathrm{mM} \mathrm{KCl}$, $\left.1 \mathrm{mM}, \mathrm{CaCl}_{2}\right)$. Glucose uptake was measured as described previously (27) using $2-\left[{ }^{3} \mathrm{H}\right]$ deoxyglucose. Each condition was assayed in triplicate.

Statistical analysis. Statistical analysis was performed using the ANOVA test (Fisher, multiple comparisons).

\section{Results}

Recruitment of intracellular GLUT4 into remodeled actin filaments. We previously reported that upon insulin stimulation, a subpopulation of intracellular GLUT4-containing vesicles colocalize with remodeled actin filaments in L6 myotubes (12). To further investigate the morphology of the actin structures that result from insulin stimulation, optical sections of $0.67 \mu \mathrm{m}$ running from the ventral to the dorsal cell surface were examined using laser scanning confocal microscopy in L6 myotubes double stained for F-actin and GLUT4myc. From the perinuclear region upward, the staining pattern of F-actin reorganized into a meshlike structure (Figure 1a, white arrows) that extended to the dorsal cell surface at selective loci. The effect of insulin on the distribution of GLUT4-myc in the same cell is shown in Figure $1 \mathrm{~b}$. Toward the dorsal surface of the cell, a portion of GLUT4-myc showed a staining pattern that mimicked the morphology of the actin-rich structures (colocalization; Figure 1a, white arrows). Near the ventral surface, even after insulin stimulation, a large portion of the actin filaments remained in the form of stress fibers and a significant portion of GLUT4-myc remained perinuclear, as in the basal state. These results are consistent with quantitations of the distribution of GLUT4myc in L6 myoblasts, showing that 90\% of GLUT4myc is intracellularly located in the basal state and that insulin causes a doubling of the surface GLUT4myc levels, still leaving about $80 \%$ of GLUT 4 in intracellular compartments (28). Therefore, in L6 muscle cells, as in 3T3-L1 adipocytes, it is not easy to see a diminution of the intracellular GLUT4 pool in response to insulin.

It is often considered that actin structures support deformabilites of the cell surface, i.e., membrane ruf- 


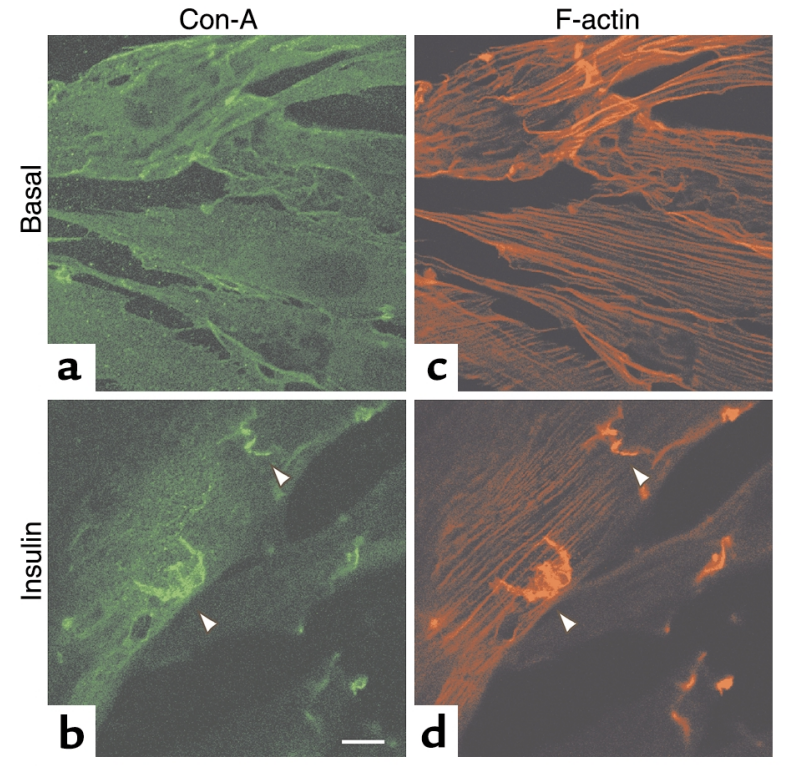

fles. To examine whether the cell surface was remodeled in areas where actin reorganized, a fluorescently tagged lectin, concanavalin-A (FITC-Con-A), was used to label the cell surface followed by cell permeabilization and labeling of F-actin (rhodamine-phalloidin). In the basal state, Con-A was evenly distributed across the myotube surface (Figure 2a). Upon insulin stimulation, a marked correspondence was observed between the localization of actin structures (Figure $2 \mathrm{~d})$ and the membrane ruffles (Figure $2 \mathrm{~b}$ ).

Cell-surface GLUT4 molecules are enriched at sites of membrane ruffle formation. We next examined whether the insertion of GLUT4 into the cell surface occurs at or near portions of the membrane that are distorted by cortical cytoskeletal reorganization. The presence of the tag c-myc epitope in the first extracellular loop of GLUT4 allows the direct detection of GLUT4 insertion into the plasma membrane in unpermeabilized myotubes by cell-surface immunofluorescence of GLUT4myc and phase contrast resolution of mem-

\section{Figure 2}

Dorsal actin-rich structures cause the membrane ruffling in L6 myotubes. Serum-deprived L6 GLUT4myc myotubes were left untreated (a and $\mathbf{c}$ ) or stimulated with $100 \mathrm{nM}$ insulin for 10 minutes (b and $\mathbf{d}$ ) at $37^{\circ} \mathrm{C}$. The plasma membrane surface was then stained using FITC-conjugated Con-A $\left(25 \mu \mathrm{g} / \mathrm{mL}\right.$ for 30 minutes at $\left.4^{\circ} \mathrm{C}\right)$, followed by permeabilization and staining for $\mathrm{F}$-actin using rhodamine-phalloidin, as described in Methods. Scale bar $=10 \mu \mathrm{m}$. Arrows indicate regions of membrane ruffling. The images are representative of three experiments.

branes ruffles, as well as by immunogold labeling of GLUT4myc and backscatter electron microscopy.

The pattern of distortion of the cell surface observed by phase contrast microscopy (Figure 3a) closely resembled the membrane ruffles detected in Figure 2. Immunostaining of the myc epitope on GLUT4 at the cell surface demonstrated a close spatial correlation between membrane ruffles and the appearance of GLUT4myc protein after insulin stimulation (Figure 3, $\mathrm{b}$ and $\mathrm{c}$ ). These findings suggest that insulin-induced actin remodeling is spatially linked to the incorporation of GLUT4 vesicles into the plasma membrane. However, the resolution of confocal fluorescence microscopy does not allow differentiation of whether the higher GLUT4myc signal arises from a larger amount of membrane sampled in the ruffled areas.

To resolve between these possibilities, the ultrastructural relationship between surface GLUT4myc protein and membrane ruffles was examined. To this end, we used the powerful approach of combined backscatter scanning electron microscopy (29). Myc epitopes on the surface of L6 myotubes were labeled immunologically with anti-myc antibody followed by secondary antibody coupled to colloidal 30-nm-diameter gold particles. Cells were prepared for scanning electron microscopy as described in Methods. On examination of these specimen on the scanning electron microscope, electrons are scattered back by the gold particles. The backscatter imaging mode of the microscope provides an intensity map of the backscattered electron yield from the specimen. Simultaneously, the micro-
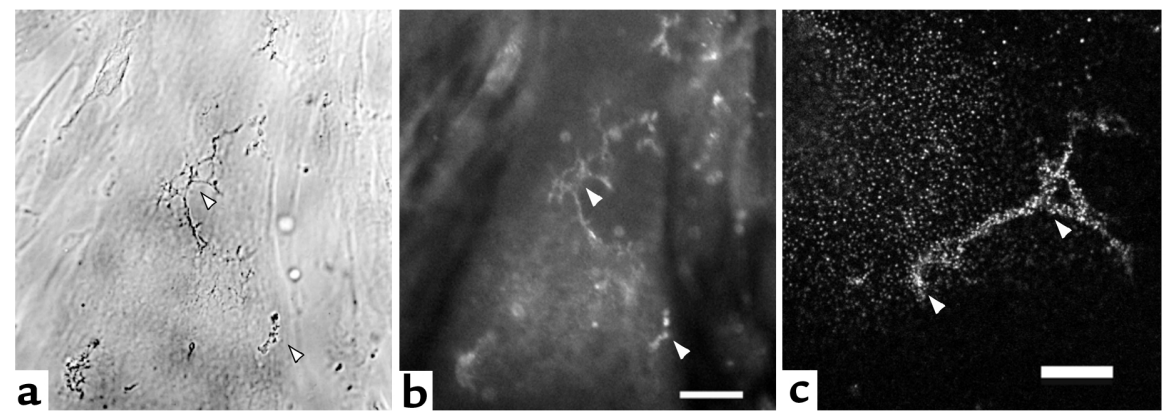

\section{Figure 3}

The pattern of insulin-induced membrane ruffling parallels the immunostaining of GLUT4myc on the surface of nonpermeabilized myotubes. Serum-deprived L6 GLUT4myc myotubes were stimulated with $100 \mathrm{nM}$ insulin for 10 minutes at $37^{\circ} \mathrm{C}$, then stained with anti-myc antibody followed by FITC-conjugated goat anti-mouse antibody to detect cell-surface GLUT4myc, as described in Methods. (a) Phase contrast image of a region of the cell pictured in $\mathbf{b}$ that is immunostained for cell-surface GLUT4myc. Scale bar $=20 \mu \mathrm{m}$. (c) Confocal image of cell-surface GLUT4myc staining in the membrane protrusions of a different cell at higher magnification. Scale bar $=7.5 \mu \mathrm{m}$. Arrows indicate regions of membrane ruffling. The images are representative of four experiments. 
a

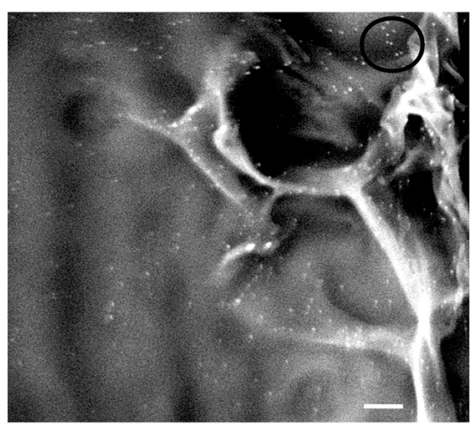

b

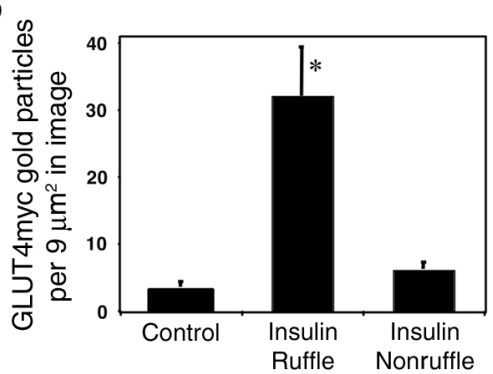

contours of the surface are imaged by using the surface scanning mode (secondary electron imaging). Upon mixing the backscattered electron signal with the secondary electron signal, a high-resolution topographical image of cell-surface GLUT4myc labeled with colloidal gold particles is obtained (29). Backscattered electron images reveal all the gold particles as white dots and enable quantitation of the number of goldlabeled epitopes on the cell surface. In unstimulated cells, the cell surface was smooth; insulin treatment caused a gradual distortion of the dorsal membrane, noticeable after 3 minutes and fully developed by 10 minutes. Figure $4 \mathrm{a}$ shows the membrane topography of a myotube stimulated with insulin for 15 minutes, and the density of white dots (colloidal gold particles detecting GLUT4myc) in both the ruffled and flatter areas of the surface. It is apparent that gold particles are more often associated with the ruffled areas than the more distant, flatter regions. Four experiments of control and insulin-stimulated cells were performed, and the density of gold particles was quantitated as described in Methods (Figure $4 b$ ). In regions rich in membrane ruffles, there was a higher gain in GLUT4myc gold signal (tenfold, relative to control cells) than in nonruffled areas (twofold).

Association of SNARE proteins with membrane ruffles. Given the close association between GLUT4myc appearance on the cell surface and regions of membrane ruffling, we next examined the spatial relationship between actin filaments and the target SNARE proteins syntaxin 4 and SNAP-23. Both of these SNARE proteins reside in the plasma membrane and have previously been implicated in the fusion of GLUT4 vesicles with the cell surface (reviewed in ref. 30). L6 myotubes were left untreated or stimulated for 10 minutes with $100 \mathrm{nM}$ insulin;

\section{Figure 5}

The t-SNAREs syntaxin4 and SNAP-23 become concentrated in the insulininduced membrane ruffles. Serumdeprived L6 GLUT4myc myotubes were stimulated with (d-f and $\mathbf{j}-\mathbf{I})$ or without (a-c and $\mathbf{g}-\mathbf{i}$ ) $100 \mathrm{nM}$ insulin for $10 \mathrm{~min}$ utes at $37^{\circ} \mathrm{C}$, followed by fixation and permeabilization. Actin was labeled with Oregon Green-conjugated phalloidin. Syntaxin4 (a-f) and SNAP-23 (g-I) were stained with specific polyclonal antibodies, followed by Cy3-conjugated secondary antibody as described in Methods. Composite images of F-actin and t-SNARE protein staining are presented in panels $\mathbf{c}, \mathbf{f}, \mathbf{i}$, and $\mathbf{I}$. Arrows indicate regions of remodeled actin filaments. The figure illustrates the diverse appearance of the dorsal actin structures depending on their three-dimensional nature and the direction of the optical section. The images are representative of three experiments. Scale bar $=10 \mu \mathrm{m}$.
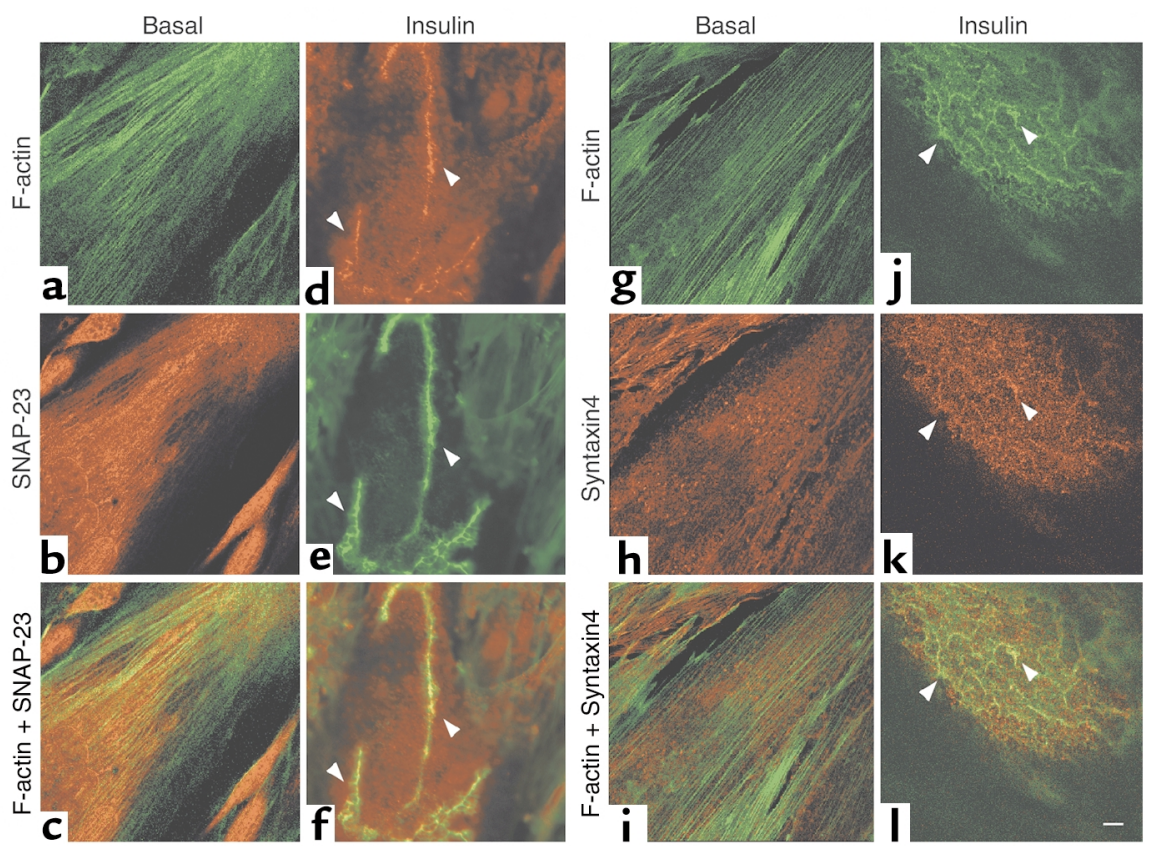


\section{Figure 6}

The $v$-SNARE VAMP2, but not VAMP3, is found in the insulininduced remodeled actin. Serumdeprived L6 GLUT4myc myotubes were stimulated with ( $\mathbf{d}-\mathbf{f}$ and $\mathbf{j}-\mathbf{I})$ or without (a-c and $\mathbf{g}-\mathbf{i}$ ) $100 \mathrm{nM}$ insulin for 10 minutes at $37^{\circ} \mathrm{C}$, followed by fixation and permeabilization. Actin was labeled with Oregon Green-conjugated phalloidin. VAMP2 (a-f) or VAMP3 (g-I) proteins were stained with specific polycolonal antibodies, followed by Cy3-conjugated secondary antibody, as described in Methods. Composite images of F-actin and $v$-SNARE protein staining are presented in panels $\mathbf{c}, \mathbf{f}, \mathbf{i}$, and $\mathbf{I}$. Arrows indicate regions of remodeled actin filaments. The images are representative of three experiments. Scale bar $=10 \mu \mathrm{m}$.
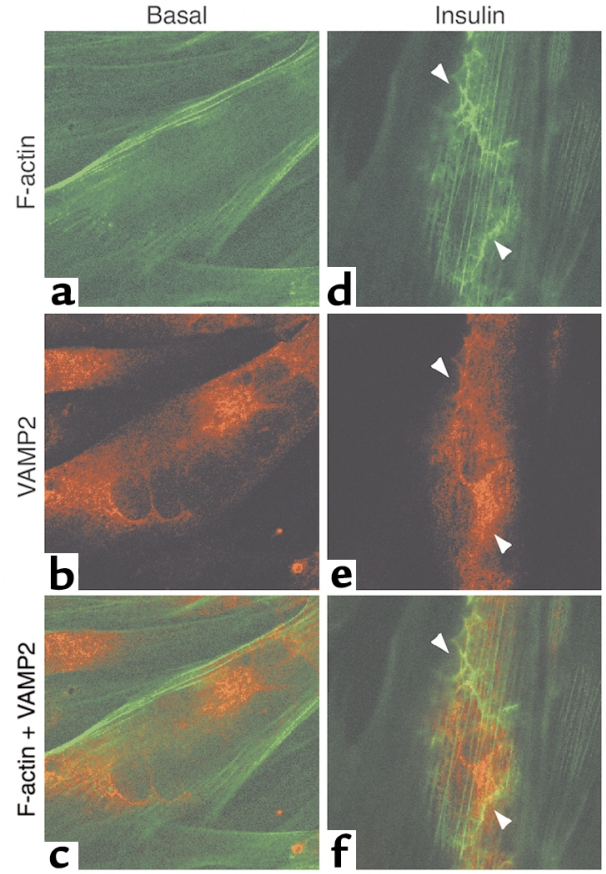
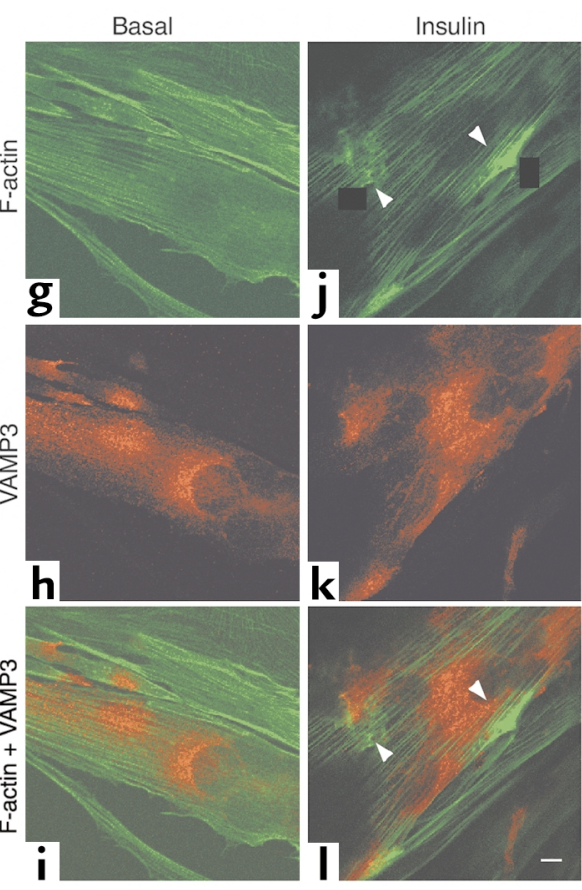

subsequently, the distribution of endogenous syntaxin 4 or SNAP-23 and filamentous actin was examined by confocal microscopy. In the basal state, a portion of SNAP-23 appeared to be filamentous and already partially colocalized with actin filaments below the cell surface, running longitudinally along the myotube axis (Figure 5b). In contrast, syntaxin 4 was diffusely distributed at the cell membrane and did not colocalize with actin stress fibers in the basal state (Figure $5 \mathrm{~h}$ ). Upon insulin treatment, both syntaxin 4 and SNAP-23 staining assumed a pattern in an optical section focused at the dorsal myotube surface. This distribution partially corresponded to the newly formed dorsal actin-rich structures supporting membrane ruffles (correspondence indicated by the white arrows in Figure 5, f and l).

We next examined whether vesicles containing the v-SNAREs VAMP2 and VAMP3 are drawn into the cortical actin structures induced by insulin, as GLUT4 is known to colocalize with a subset of these v-SNARE proteins (reviewed in ref. 30). L6 myotubes were left untreated or stimulated with insulin, and subsequently endogenous VAMP2 or VAMP3 as well as filamentous actin were labeled with specific antibodies or phalloidin, respectively. Under unstimulated conditions, both v-SNAREs were localized to a perinuclear region, which was devoid of actin filament staining (Figure 6, $\mathrm{b}$ and $\mathrm{h})$. Upon insulin treatment $(100 \mathrm{nM}, 10 \mathrm{~min}-$ utes), a striking difference in VAMP localization was observed: whereas a distinct VAMP2 signal was detected in the subcortical actin structures (Figure 6, e and f, white arrows), VAMP3 clearly escaped such relocalization (Figure 6, $\mathrm{k}$ and $\mathrm{l}$ ).

Interfering with actin remodeling reduces GLUT4 translocation. An intact actin cytoskeleton is required for insulin-mediated glucose transport in L6 myotubes (6), 3T3-L1 adipocytes (8), and rat adipocytes (13). The drugs used to disrupt actin polymerization in those studies (cytochalasin-D, latrunculin-B) result in complete dispersal of filamentous actin $(31,32)$, making it difficult to conclude whether it is the existence of actin filaments or the remodeling of those filaments that is required for insulin-regulated GLUT4 traffic. We therefore compared two chemical agents: jasplakinolide, which prevents actin turnover by preventing depolymerization, leading to a dispersal of intact actin (33), and swinholide-A, which selectively interferes with actin filament remodeling, leaving the normal cytoskeleton intact (34).

The marine sponge peptide jasplakinolide stabilizes actin filaments by binding to both barbed and pointed ends, thus preventing their dynamic turnover (33). L6 GLUT4myc myotubes were preincubated with jasplakinolide for 30 minutes before treatment with insulin for 10 minutes. To ensure that jasplakinolide treatment resulted in depolymerization of actin filaments, we first examined the effect of the agent on actin morphology in L6 myotubes. Because jasplakinolide occupies the same binding site on filamentous actin as phalloidin (33), we detected F-actin using a $\beta$-actin specific antibody. As shown in Figure $7, a-d$, $\beta$-actin antibodies stained the actin stress fibers that run longitudinally along the myotube axis in the basal state (Figure 7a) and which were remodeled upon insulin stimulation (Figure 7b). Jasplakinolide caused a shortening and thickening of basal state actin fibers (Figure 7c) that could no longer remodel into a mesh after insulin stimulation (Figure 7d).

The effect of disrupting actin filaments with jasplakinolide on the insertion of GLUT4myc molecules 


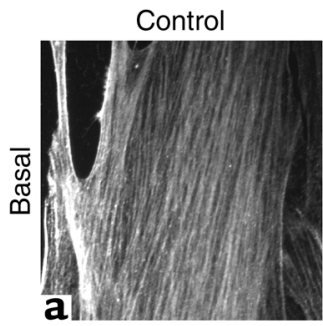

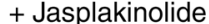

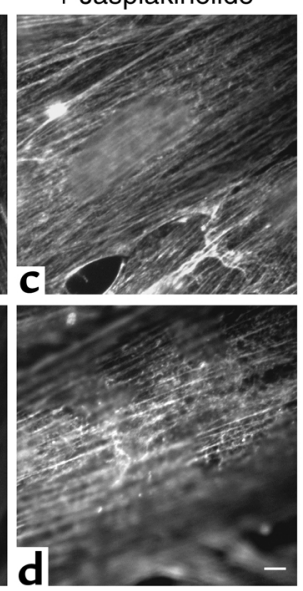

+ Jasplakinolide

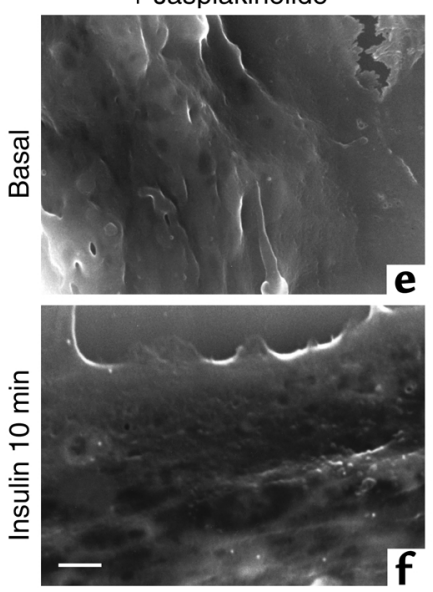

g

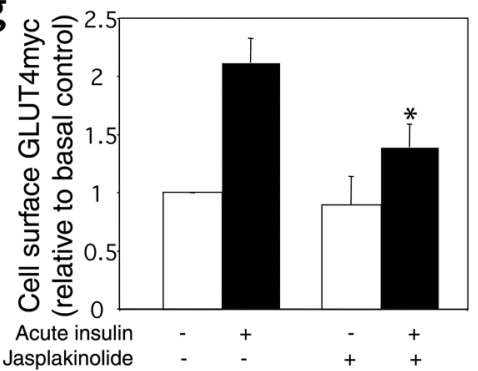

\section{Figure 7}

Jasplakinolide disrupts actin filament turnover and reduces insulin-induced actin remodeling and GLUT4 translocation. L6 GLUT4myc myotubes were exposed to $2 \mu \mathrm{M}$ jasplakinolide for the final 30 minutes of serum deprivation and were then treated with or without $100 \mathrm{nM}$ insulin for 10 minutes $(\mathbf{a}-\mathbf{f})$ or 30 minutes $(\mathbf{g})$ at $37^{\circ} \mathrm{C}$. (a-d) Cells were fixed and stained for actin using mAb against $\beta$-actin coupled to FITC-conjugated goat anti-mouse antibody. The images are representative of three experiments. Scale bar $=10 \mu \mathrm{m}$. (e and $\mathbf{f})$ Cell-surface GLUT4myc was labeled with gold particles, and composite signals of the scanning electron mode and the backscattered electrons from the gold particles on the cell surface were obtained as described in Figure 3. (Examples of basal and insulin cells in the absence of jasplakinolide are shown in Figure 3a.) Scale bar $=1 \mu \mathrm{m}$. (g) Densitometric analysis of cell-surface GLUT4myc was quantified after treatment of myotubes with or without $100 \mathrm{nM}$ insulin for 30 minutes, as described in Methods. Results in $\mathrm{g}$ are expressed as the fold change in cell-surface GLUT4myc relative to basal cells in the untreated control (mean \pm SEM of three independently performed experiments). ${ }^{*} P<0.05$ compared with respective control in the absence of jasplakinolide.

into plasma membrane ruffles was examined by scanning electron microscopy coupled to backscatter detection of immunogold-labeled anti-myc antibody in nonpermeabilized cells (Figure 7, e and f). Preincubation with jasplakinolide caused a slight distortion of the myotube cell surface (Figure 7e) and fully prevented insulin-induced formation of membrane ruffles (Figure 7f). The increased density of cell-surface gold-labeled GLUT4myc caused by insulin was also markedly reduced by this drug (Figure 7f). A quantitation of the levels of GLUT4myc on the cell surface detected by a densitometric assay is presented in Figure $7 \mathrm{~g}$. The increase in cell-surface GLUT4myc levels induced by insulin was reduced by $71 \%(P<0.05)$ by jasplakinolide treatment.

The macrolide swinholide-A prevents actin subunits from participating in actin-filament nucleation or elongation reactions (34), thereby preventing stimulusinduced cortical actin remodeling, without affecting the intact actin network. Unlike jasplakinolide (Figure 7, c and d), pretreatment of L6 myotubes with swinholideA did not affect the intensity of F-actin stress fibers in the basal state (Figure 7a). Importantly, when swinholide-A-treated cells were stimulated with insulin, an incomplete formation of the actin mesh was observed (Figure 8b). The resulting actin structures were shorter and contained significantly less branching (Figure 8b). The correlation between the morphological and metabolic consequences of partial cortical actin remodeling was further confirmed by the densitometric quantitation of surface GLUT4myc in insulin-stimulated cells pretreated with swinholide-A. Interestingly, swinholideA treatment reduced $(62 \%, P<0.05)$ GLUT4myc appearance at the cell surface (Figure 8c) after insulin stimulation, similar to the partial inhibition of actin remodeling observed in Figure 8.

High glucose/insulin causes insulin resistance of actin remodeling and GLUT4 translocation. The observation that insulin partially remodeled actin filaments in cells pretreated with swinholide-A and caused only $38 \%$ mobilization of intracellular GLUT4 to the plasma membrane indicates that swinholide-A induced a form of "resistance" of L6 cells to insulin. Importantly, there is ample evidence that GLUT4 externalization is defective in the pathophysiological state of insulin resistance underlying type 2 diabetes (5). Therefore, we examined the possibility that strategies that render cells insulin resistant might affect actin remodeling and GLUT4 delivery to the membrane. For this purpose, we tested the effect of prolonged preexposure to high glucose and insulin, a strategy shown to cause insulin resistance in fat cells (35). L6 myotubes were exposed to high insulin $(100 \mathrm{nM})$ and high glucose $(25 \mathrm{mM})$ for 24 hours. Chronic exposure of L6 cells to high glucose and insulin reduced the insulin stimulation of glucose uptake in L6 myotubes significantly: Glucose uptake increased only by $3.14 \pm 0.27 \mathrm{pmol} / \mathrm{min} / \mathrm{mg}$ protein above the basal value, compared with $6.93 \pm 0.19 \mathrm{pmol} / \mathrm{min} / \mathrm{mg}$ protein in control cells. Insulin caused only a 1.4-fold increase in glucose uptake relative to the basal value in the challenged cells, compared with 1.9-fold in control cells. Hence, insulin resistance of glucose uptake arises upon 

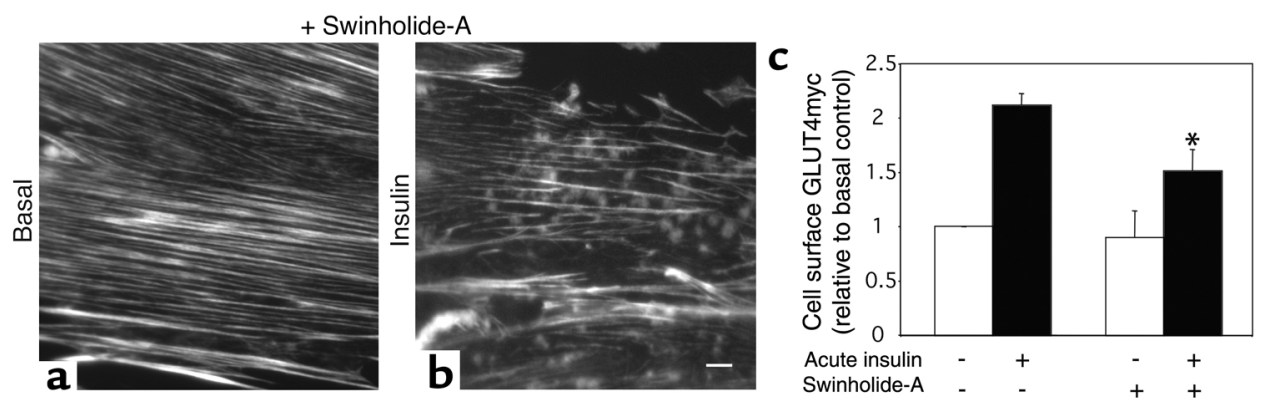

\begin{abstract}
Figure 8
Swinholide-A selectively blocks actin remodeling and partially reduces insulin-stimulated GLUT4 translocation. L6 GLUT4myc myotubes were exposed to $12.5 \mathrm{nM}$ swinholide-A for the final hour of serum deprivation and were then treated with or without $100 \mathrm{nM}$ insulin for 10 minutes (a and $\mathbf{b}$ ) or 30 minutes (c) at $37^{\circ} \mathrm{C}$. (a and $\mathbf{b}$ ) Cells were fixed and stained for F-actin using Oregon Green-phalloidin as described in Figure 1. The images are representative of four experiments. Scale bar $=10 \mu \mathrm{m}$. (c) Quantitation of cell-surface GLUT4myc was determined after treatment of the myotubes with or without $100 \mathrm{nM}$ insulin for 30 minutes, as described in Methods. Results in c are expressed as the fold change in cell-surface GLUT4myc relative to basal cells in the untreated control (mean \pm SEM of three independently performed experiments). ${ }^{*} P<0.05$ compared with respective control in the absence of swinholide-A.
\end{abstract}

exposing cells with high glucose and insulin for prolonged times. Interestingly, in this milieu, insulininduced recruitment of GLUT4myc to the plasma membrane was reduced by $45 \%$ (Figure $9 \mathrm{a}$ ), reflecting the insulin resistance of glucose uptake. The high glucose/insulin challenge also affected the amount of GLUT4myc transporters in the basal state. The insulindependent gain in GLUT4myc was reduced from 1.1 relative units in control cells to 0.6 units in challenged cells. These observations suggest that both the insulindependent and the constitutive recycling of this protein to/from the plasma membrane were altered.

Strikingly, prolonged exposure to high glucose and insulin also lead to aberrant actin mesh formation in insulin-treated cells (Figure 9e), reminiscent of the partial actin remodeling observed in swinholide-A-treated cells (Figure $8 \mathrm{~b}$ ). Approximately $80 \%$ of myotubes normally display dense actin-rich structures and membrane ruffles when acutely challenged with insulin. In cells chronically incubated with high glucose/insulin, approximately $30 \%$ of the myotubes formed actin structures, and these were discrete and scattered (Figure 9e) compared with the actin structures in control insulin-sensitive cells (Figure 9c).

\section{Discussion}

In addition to its well-characterized metabolic effects, insulin induces remodeling of actin filaments below the plasma membrane which leads to the formation of membrane ruffles (9). Although participation of the actin cytoskeleton in insulin-stimulated GLUT4 traffic has been established $(6,8,13)$, the exact function of insulin-dependent cortical actin remodeling is not fully understood. Furthermore, the possibility that an imposed state of insulin resistance could alter actin filament remodeling has not been explored. We previously showed that a portion of the cellular content of the insulin signaling molecule PI3-K (p85 subunit) as well as GLUT4 vesicles relocate toward the insulin-induced actin-rich structures (12). Here we show that a subset of GLUT4 vesicles are inserted into sites of membrane ruffling caused by actin remodeling. These membrane ruffle sites appear to concentrate the t-SNAREs syntaxin4 and SNAP-23 and the v-SNARE VAMP2. Moreover, we provide evidence suggesting that a diabetic-mimicking milieu leads to a defect in cortical actin remodeling and this defect may contribute to the impairment in GLUT4 translocation to the cell surface.

Insulin-induced actin remodeling and GLUT4 translocation. Z-scan sectioning across the depth of L6 myotubes performed in Figure 1 revealed the spatial relationship between GLUT4-containing vesicles and the insulin-induced actin structures. A portion of the intracellular (perinuclear) GLUT4 became concentrated in the newly formed actin-rich structures at the dorsal surface of the myotube. These observations suggest that actin filaments may directly participate in intracellular traffic of GLUT4-containing vesicles. In addition to GLUT4, GLUT1 and GLUT3 are also expressed in parental L6 myotubes (36). L6 GLUT4myc myotubes express more than ten times higher levels of GLUT4myc than do any of the endogenous transporters. GLUT3 is largely on the cell surface, whereas half of the GLUT1 is intracellular and responds to insulin (36). The intracellular GLUT1 segregates only partially with immunopurified GLUT4-containing intracellular compartments in L6 GLUT4myc cells (37). An in-depth study on the relationship between GLUT1 and the cytoskeleton is currently under way. The presence of an exofacial c-myc epitope tag on GLUT4 enabled us to identify the site of incorporation of the protein into the plasma membrane. Using scanning electron microscopy coupled to immunogold labeling of the c-myc epitope, we provide ultrastructural evidence suggesting that translocation and fusion of GLUT4 may preferentially occur in ruffled regions of the membrane. Indeed, a quantitatively higher GLUT4myc immunogold signal was detected in the ruf- 


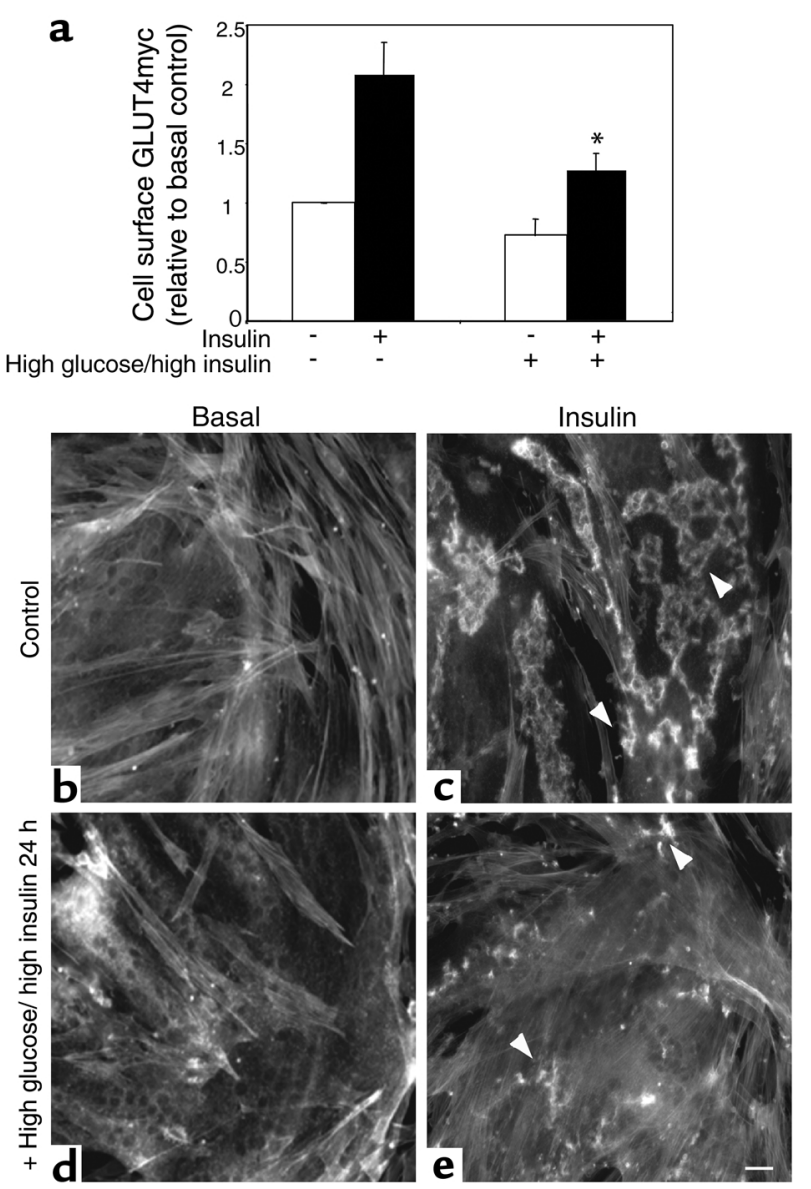

Figure 9

Insulin resistance caused by chronic high glucose and insulin impairs insulin-stimulated, GLUT4 externalization and actin remodeling. L6 GLUT4myc myotubes were maintained in high insulin (100 nM) and high glucose (25 mM) for 24 hours before serum deprivation for 4 hours. Myotubes were then treated with or without $100 \mathrm{nM}$ insulin for 30 minutes (a) or 10 minutes (b-e) at $37^{\circ} \mathrm{C}$. (a) Quantification of cellsurface GLUT4myc was performed, as described in Methods. Results are expressed as the fold change relative to basal cells in the untreated control (mean \pm SEM of four independently performed experiments). ${ }^{*} P<0.05$ compared with respective control in the absence of high insulin/high glucose. (b-e) Cells were fixed and stained for F-actin using Oregon Green-phalloidin as described in Figure 1. Arrows indicate regions of remodeled (c) or partially remodeled (e) actin filaments. The images are representative of four experiments. Scale bar $=50 \mu \mathrm{m}$.

fles compared with nonruffled regions of the cell surface of insulin-stimulated cells (Figure 4). Immunofluorescence of nonpermeabilized cells confirmed that surface GLUT4 proteins spatially correspond to areas of ruffles (Figure 3). The observation that the FITC-ConA signal was also stronger in the ruffles suggests that there is indeed more membrane area in those regions. It is tempting to speculate that insulin-dependent formation of cortical actin structures may target the ongoing fusion of a subgroup of intracellular GLUT4 vesicles with the plasma membrane and that this contributes to the expansion of the membrane, allowing the formation of membrane ruffles. Our findings are consistent with other recent observations that GLUT4-containing vesi- cles are targeted to and fuse at specific loci on the plasma membrane of fat cells $(15,38)$.

Relationship of SNARE proteins to actin structures. Three non-neuronal SNARE proteins have been implicated in the fusion of GLUT4-containing vesicles with the plasma membrane after insulin treatment in muscle cells and adipocytes. Unlike VAMP2, VAMP3/cellubrevin is not required for insulin-dependent GLUT4 vesicle fusion (19), although both proteins have been reported to coexist on GLUT4-containing vesicles (17, 24, 39-41). Their cognate t-SNARE proteins SNAP-23 and syntaxin 4 are present in the plasma membrane $(20,42)$, where SNAP-23 mediates the formation of a complex between syntaxin 4 and VAMP2 that is required for the incorporation of GLUT4 into the plasma membrane of 3T3-L1 adipocytes $(17,20,22,23)$. It is conceivable that remodeling of actin filaments may facilitate the interaction between plasma membrane t-SNAREs and v-SNAREs on the GLUT4 vesicles. In keeping with this possibility, SNAP-23 sediments with detergent-insoluble material containing cytoskeletal elements (22). We therefore explored whether insulin-induced actin remodeling could modulate the spatial distribution of the t-SNAREs syntaxin 4 and SNAP-23. Under basal conditions, syntaxin 4 was located preferentially at the surface membrane, where it presented a diffuse distribution and did not show any colocalization with F-actin (Figure $5, \mathrm{~h}-\mathrm{i}$ ). In contrast, in the basal state, SNAP-23 appeared as filamentous structures within the cell and at the cell surface, and it partially colocalized with F-actin stress fibers (Figure 5, b and c). After insulin stimulation, a portion of both SNAP-23 and syntaxin 4 became prominent in the membrane ruffles. The presence of t-SNARE proteins and GLUT4 vesicles in membrane ruffles may enable efficient interactions between t-SNAREs and v-SNAREs on the GLUT4 compartment, leading to fusion of GLUT4-containing vesicles with the plasma membrane. We therefore examined the cellular distribution of the v-SNARE proteins VAMP2 and VAMP3/cellubrevin by fluorescence microscopy. In myotubes stimulated with insulin, a portion of VAMP2 colocalized with the actin structures, whereas immunostaining for VAMP3 continued to be diffuse and perinuclear. These results are consistent with the notion that VAMP2 and VAMP3 populate distinct vesicles and that only insulin-responsive GLUT4 membranes containing VAMP2 gather into the actin mesh in response to insulin. We suggest that SNAP-23 and syntaxin 4 in the membrane ruffles may serve as anchoring sites for the incoming VAMP2-containing GLUT4 vesicles gathered by the cortical actin mesh.

Impairment of insulin action by perturbation of actin filaments. Interfering with actin turnover by jasplakinolide inhibited $71 \%$ of insulin-mediated GLUT4myc translocation to the cell surface (Figure 7). This result is consistent with previous findings reported with cytochalasin$\mathrm{D}$ and latrunculin-B, two unrelated compounds that lead to actin filament depolymerization $(6,8,13,26,15)$. Unlike all of these other agents, swinholide-A selectively 
interferes with the dynamic turnover of cortical actin yet maintains an intact network of actin stress fibers. Swinholide-A pretreatment reduced the formation of actin structures in response to insulin (Figure $8 \mathrm{~b}$ ). In parallel, it caused a reduction $(62 \%)$ in the redistribution of GLUT4 to the cell surface (Figure 8c), indicating that the remodeling of actin filaments is required for GLUT4 externalization, and not merely an intact actin network. Prolonged preexposure of L6 myotubes to conditions of insulin resistance (high glucose and insulin) diminished insulin-stimulated glucose uptake and GLUT4myc translocation. The effect of the high glucose/insulin milieu was strikingly similar to that caused by swinholide-A on actin filament reorganization and GLUT4myc translocation. These observations raise the question of which events might be sensitive to both swinholide-A and high glucose/high insulin. Recent evidence points to the following three potential cytoskeleton-dependent steps in the insulin signaling pathway.

(a) Coupling of the insulin receptor with its downstream effectors. It was recently observed that prolonged exposure of 3T3-L1 adipocytes to high insulin releases insulin receptor substrate (IRS) from a cytoskeletal complex, leading to an inability of the insulin signal to propagate beyond the insulin receptor (43).

(b) The communication of PI3-K with its target signaling molecules. We previously showed that the actin cytoskeleton appears to concentrate PI3-K in specific cellular compartments in L6 myotubes (12) and 3T3$\mathrm{L} 1$ adipocytes (8). Moreover, it was recently reported that actin filament disruption prevents the generation of 3-phosphoinositides and Akt/protein kinase B (PKB) activation (44). Thus, the inhibition of cortical actin remodeling may limit the access of PI3-K products to their specific downstream targets, important for insulin-stimulated GLUT4 translocation. This possibility is supported by recent reports of impaired signaling downstream of PI3-K in various cellular and animal models of insulin resistance $(45,46)$.

(c) The arrival and fusion of GLUT4 vesicles at the plasma membrane. There have been several recent reports of microtubule and intermediate filament cytoskeletal networks serving as tracks for GLUT4-containing endomembranes $(14,15,47-49)$. Given the intimate interplay between actin and the other cytoskeletal networks (50), it is possible that disturbance of either cytoskeleton during insulin resistance may cause a coordinate disruption of all three networks, leading to inappropriate targeting of GLUT4-containing compartments. It is conceivable that the incompletely formed cytoskeletal architecture may no longer be capable of providing the scaffolding for $v$-SNAREs on the GLUT4-containing vesicles to appose t-SNAREs in ruffled regions of the cell surface in response to insulin.

Future studies will be required to elucidate which of these mechanisms participate in the swinholide-A- and high glucose and insulin-induced insulin resistance.

In summary, insulin-mediated actin reorganization modulates the redistribution of GLUT4-containing vesi- cles and SNARE proteins to a specific cellular location below the cell surface. We propose that the newly formed actin-rich structures provide the scaffold whereby signaling molecules, GLUT4-containing vesicles, and SNARE proteins interact to facilitate the insertion of GLUT4 to selective loci (ruffles) in the plasma membrane. We further propose that the integrity of the actin cytoskeleton is essential for the full insulin-induced translocation of GLUT4 to the cell surface, and that partial remodeling of F-actin causes insulin resistance of actin remodeling and GLUT4 externalization. The actindependent compartmentalization of signaling molecules, GLUT4 vesicles, and the SNARE fusion machinery may contribute to the metabolic actions of insulin in target tissues such as skeletal muscle and may be defective in pathological conditions such as insulin resistance.

\section{Acknowledgments}

We are grateful to Cameron Ackerley and Aina Tilups (Department of Pathobiology and Laboratory Medicine, Hospital for Sick Children) for expert advice and assistance with electron microscopy techniques used in this study. This study was supported by a grant from the Canadian Institutes of Health Research (MT7307) to A. Klip. P. Tong was supported by a traveling fellowship from the Peels Medical Research Trust. Z.A. Khayat was supported by a Canadian Institutes of Health Research Doctoral Student Award.

1. Douen, A.G., et al. 1990. Exercise induces recruitment of the "insulin-responsive glucose transporter". Evidence for distinct intracellular insulin- and exercise-recruitable transporter pools in skeletal muscle. J. Biol. Chem. 265:13427-13430.

2. Hirshman, M.F., Goodyear, L.J., Wardzala, L.J., Horton, E.D., and Horton, E.S. 1990. Identification of an intracellular pool of glucose transporters from basal and insulin-stimulated rat skeletal muscle. J. Biol. Chem. 265:987-991.

3. Rothman, D.L., et al. 1995. Decreased muscle glucose transport/phosphorylation is an early defect in the pathogenesis of non-insulin-dependent diabetes mellitus. Proc. Natl. Acad. Sci. USA. 92:983-987.

4. Zierath, J.R., et al. 1996. Insulin action on glucose transport and plasma membrane GLUT4 content in skeletal muscle from patients with NIDDM. Diabetologia. 39:1180-1189.

5. Zierath, J.R., Krook, A., and Wallberg-Henriksson, H. 2000. Insulin action and insulin resistance in human skeletal muscle. Diabetologia. 43:821-835.

6. Tsakiridis, T., Vranic, M., and Klip, A. 1994. Disassembly of the actin network inhibits insulin-dependent stimulation of glucose transport and prevents recruitment of glucose transporters to the plasma membrane. J. Biol. Chem. 269:29934-29942.

7. Vollenweider, P., et al. 1997. The small guanosine triphosphate-binding protein Rab4 is involved in insulin-induced GLUT4 translocation and actin filament rearrangement in 3T3-L1 cells. Endocrinology. 138:4941-4949.

8. Wang, Q.H., Bilan, P.J., Tsakiridis, T., Hinek, A., and Klip, A. 1998. Actin filaments participate in the relocalization of phosphatidylinositol 3-kinase to glucose transporter-containing compartments and in the stimulation of glucose uptake in 3T3-L1 adipocytes. Biochem. J. 331:917-928.

9. Ridley, A.J., Paterson, H.F., Johnston, C.L., Diekmann, D., and Hall, A. 1992. The small GTP-binding protein rac regulates growth factor-induced membrane ruffling. Cell. 70:401-410.

10. Knight, J.B., Yamauchi, K., and Pessin, J.E. 1995. Divergent insulin and platelet-derived growth factor regulation of focal adhesion kinase (pp125FAK) tyrosine phosphorylation, and rearrangement of actin stress fibers. J. Biol. Chem. 270:10199-10203.

11. Clodi, M., et al. 1998. Effects of general receptor for phosphoinositides 1 on insulin and insulin-like growth factor I-induced cytoskeletal rearrangement, glucose transporter- 4 translocation, and deoxyribonucleic acid synthesis. Endocrinology. 139:4984-4990.

12. Khayat, Z.A., Tong, P., Yaworsky, K., Bloch, R.J., and Klip, A. 2000. Insulininduced actin filament remodeling colocalizes actin with phosphatidylinositol 3-kinase and GLUT4 in L6 myotubes. J. Cell Sci. 113:279-290.

13. Omata, W., Shibata, H., Li, L., Takata, K., and Kojima, I. 2000. Actin filaments play a critical role in insulin-induced exocytotic recruitment but not 
in endocytosis of GLUT4 in isolated rat adipocytes. Biochem. J. 346:321-328. 14. Emoto, M., Langille, S.E., and Czech, M.P. 2001. A role for kinesin in insulinstimulated GLUT4 glucose transporter translocation in 3T3-L1 adipocytes. J. Biol. Chem. 276:10677-10682

15. Patki, V., et al. 2001. Insulin action on GLUT4 traffic visualized in single 3T3-11 adipocytes by using ultra-fast microscopy. Mol. Biol. Cell. 12:129-141.

16. Sollner, T., et al. 1993. SNAP receptors implicated in vesicle targeting and fusion. Nature. 362:318-324.

17. Cheatham, B., et al. 1996. Insulin-stimulated translocation of GLUT4 glucose transporters requires SNARE-complex proteins. Proc. Natl. Acad. Sci. USA. 93:15169-15173.

18. Martin, L.B., Shewan, A., Millar, C.A., Gould, G.W., and James, D.E. 1998. Vesicle-associated membrane protein 2 plays a specific role in the insulindependent trafficking of the facilitative glucose transporter GLUT4 in 3T3L1 adipocytes. J. Biol. Chem. 273:1444-1452.

19. Randhawa, V.K., et al. 2000. VAMP2, but not VAMP3/cellubrevin, mediates insulin-dependent incorporation of GLUT4 into the plasma membrane of L6 myoblasts. Mol. Biol. Cell. 11:2403-2417.

20. Volchuk, A., et al. 1996. Syntaxin 4 in 3T3-L1 adipocytes: regulation by insulin and participation in insulin-dependent glucose transport. Mol. Biol. Cell. 7:1075-1082.

21. Olson, A.L., Knight, J.B., and Pessin, J.E. 1997. Syntaxin 4, VAMP2, and/or VAMP3/cellubrevin are functional target membrane and vesicle SNAP receptors for insulin-stimulated GLUT4 translocation in adipocytes. Mol. Cell. Biol. 17:2425-2435.

22. Foster, L.J., Yaworsky, K., Trimble, W.S., and Klip, A. 1999. SNAP23 promotes insulin-dependent glucose uptake in 3T3-L1 adipocytes: possible interaction with cytoskeleton. Am. J. Physiol. 276:C1108-C1114.

23. Rea, S., et al. 1998. Syndet, an adipocyte target SNARE involved in the insulin-induced translocation of GLUT4 to the cell surface. J. Biol. Chem. 273:18784-18792.

24. Volchuk, A., et al. 1995. Cellubrevin is a resident protein of insulin-sensitive GLUT4 glucose transporter vesicles in 3T3-L1 adipocytes. J. Biol. Chem. 270:8233-8240.

25. Kanai, F., et al. 1993. Direct demonstration of insulin-induced GLUT4 translocation to the surface of intact cells by insertion of a c-myc epitope into an exofacial GLUT4 domain. J. Biol. Chem. 268:14523-14526.

26. Wang, Q., Khayat, Z., Kishi, K., Ebina, Y., and Klip, A. 1998. GLUT4 translocation by insulin in intact muscle cells: detection by a fast and quantitative assay. FEBS Lett. 427:193-197.

27. Klip, A., Logan, J.W.J., and Lee, G. 1982. Hexose transport in L6 muscle cells. Kinetic properties and the number of $\left[{ }^{3} \mathrm{H}\right]$ cytochalasin B binding sites. Biochem. Biophys. Res. Commun. 687:265-280.

28. Li, D., Randhawa, V., Patel, N., Hayashi, M., and Klip, A. 2001. Hyperosmolarity reduces GLUT4 endocytosis and inreases its exocytosis from a VAMP2independent pool in $\mathrm{L} 6$ muscle cells. J. Biol. Chem. In press.

29. De Harven, E., Leung, R., and Christensen, H. 1984. A novel approach for scanning electron microscopy of colloidal gold-labeled cell surfaces. J. Cell Biol. 99:53-57.

30. Foster, L.J., and Klip, A. 2000. Mechanism and regulation of GLUT-4 vesicle fusion in muscle and fat cells. Am. J. Physiol. 279:C877-C890.

31. Cooper,J.A. 1987. Effects of cytochalasins and phalloidin on actin. J. Cell Biol. 105:1473-1478.

32. Spector, I., Shochet, N.R., Kashman, Y., and Groweiss, A. 1983. Latrunculins: novel marine toxins that disrupt microfilament organization in cultured cells. Science. 219:493-495.

33. Bubb, M.R., Senderowicz, A.M., Sausville, E.A., Duncan, K.L., and Korn, E.D.
1994. Jasplakinolide, a cytotoxic natural product, induces actin polymer ization and competitively inhibits the binding of phalloidin to F-actin. J. Biol. Chem. 269:14869-14871.

34. Bubb, M.R., Spector, I., Bershadsky, A.D., and Korn, E.D. 1995. Swinholide A is a microfilament disrupting marine toxin that stabilizes actin dimers and severs actin filaments. J. Biol. Chem. 270:3463-3466.

35. Thomson, M.J., Williams, M.G., and Frost, S.C. 1997. Development of insulin resistance in 3T3-L1 adipocytes. J. Biol. Chem. 272:7759-7764.

36. Bilan, P.J., Mitsumoto, Y., Maher, F., Simpson, I.A., and Klip, A. 1992. Detection of the GLUT3 facilitative glucose transporter in rat L6 muscle cells: regulation by cellular differentiation, insulin and insulin-like growth factor-I. Biochem. Biophys. Res. Commun. 186:1129-1137.

37. Ueyama, A., Yaworsky, K.L., Wang, Q., Ebina, Y., and Klip, A. 1999. GLUT4myc ectopic expression in L6 myoblasts generates a GLUT-4-specific pool conferring insulin sensitivity. Am. J. Physiol. 277:E572-E578.

38. Malide, D., Ramm, G., Cushman, S.W., and Slot, J.W. 2000. Immunoelectron microscopic evidence that GLUT4 translocation explains the stimulation of glucose transport in isolated rat white adipose cells. J. Cell Sci. 113:4203-4210.

39. Cain, C.C., Trimble, W.S., and Lienhard, G.E. 1992. Members of the VAMP family of synaptic vesicle proteins are components of glucose transportercontaining vesicles from rat adipocytes. J. Biol. Chem. 267:11681-11684.

40. Volchuk, A., et al. 1994. Expression of vesicle-associated membrane protein 2 (VAMP- 2)/synaptobrevin II and cellubrevin in rat skeletal muscle and in a muscle cell line. Biochem. J. 304:139-145.

41. Ramm, G., Slot, J.W., James, D.E., and Stoorvogel, W. 2000. Insulin recruits GLUT4 from specialized VAMP2-carrying vesicles as well as from the dynamic endosomal/trans-Golgi network in rat adipocytes. Mol. Biol. Cell. 11:4079-4091.

42. Timmers, K.I., et al. 1996. Identification of SNAP receptors in rat adipose cell membrane fractions and in SNARE complexes co-immunoprecipitated with epitope-tagged $\mathrm{N}$-ethylmaleimide-sensitive fusion protein. Biochem. J. 320:429-436

43. Clark, S.F., Molero, J.C., and James, D.E. 2000. Release of insulin receptor substrate proteins from an intracellular complex coincides with the development of insulin resistance. J. Biol. Chem. 275:3819-3826.

44. Peyrollier, K., et al. 2000. A role for the actin cytoskeleton in the hormonal and growth-factor-mediated activation of protein kinase B. Biochem. J. 352:617-622

45. Nawano, M., et al. 1999. Hyperglycemia impairs the insulin signaling step between PI 3-kinase and Akt/PKB activations in ZDF rat liver. Biochem. Biophys. Res. Commun. 266:252-256.

46. Tirosh, A., Potashnik, R, Bashan, N., and Rudich, A. 1999. Oxidative stress disrupts insulin-induced cellular redistribution of insulin receptor substrate1 and phosphatidylinositol 3-kinase in 3T3-L1 adipocytes. A putative cellular mechanism for impaired protein kinase B activation and GLUT4 translocation. J. Biol. Chem. 274:10595-10602.

47. Fletcher, L.M., Welsh, G.I., Oatey, P.B., and Tavare, J.M. 2000. Role for the microtubule cytoskeleton in GLUT4 vesicle trafficking and in the regulation of insulin-stimulated glucose uptake. Biochem. J. 352:267-276.

48. Guilherme, A., et al. 2000. Perinuclear localization and insulin responsiveness of GLUT4 requires cytoskeletal integrity in 3T3-L1 adipocytes. J. Biol. Chem. 275:38151-38159.

49. Olson, A.L., Trumbly, A.R., and Gibson, G.V. 2001. Insulin-mediated GLUT4 translocation is dependent on the microtubule network. J. Biol. Chem. 276:10706-10714.

50. Fuchs, E., and Yang, Y. 1999. Crossroads on cytoskeletal highways. Cell. 98:547-550. 